\title{
Submap Bathymetric SLAM using Structured Light in Underwater Environments
}

\author{
Miquel Massot-Campos and Gabriel Oliver \\ Dpt. of Mathematics and Computer Science \\ University of the Balearic Islands \\ Cra. de Valldemossa km 7.5 \\ 07122 Palma de Mallorca, Spain \\ \{miquel.massot, goliver\}@uib.cat
}

\author{
Adrian Bodenmann and Blair Thornton* \\ Institute of Industrial Science \\ University of Tokyo \\ 4-6-1 Komaba, Meguro-ku \\ Tokyo 153-8505, Japan \\ \{adrian, blair\}@iis.u-tokyo.ac.jp
}

\begin{abstract}
This article presents a bathymetric SLAM (simultaneous localization and mapping) solution for underwater vehicles by addressing the registration of point clouds gathered from single line laser-based structured light systems. While structured light can be applied to generate millimetre resolution seafloor bathymetry, the accuracy of the maps generated is typically constrained by the localization accuracy of the vehicles used. In this work, relative uncertainties in vehicle localisation are reduced by implementing bathymetric SLAM using temporally constrained submaps. We demonstrate that the method described can overcome misalignments by correcting errors in localisation and can be used to generate self-consistent high-resolution seafloor bathymetric maps.
\end{abstract}

\section{INTRODUCTION}

The use of high-resolution seafloor bathymetry is becoming increasingly routine in marine research [1], [2]. In particular, advances in high-frequency acoustics and optical methods combined with the use of underwater vehicles such as AUVs and ROVs allow us to map vast expanses of the seafloor at resolutions of several tens of centimetres [3].

While the instruments used to generate seafloor maps have significantly increased the spatial resolution of bathymetric maps, this improvement is only meaningful if it can be matched by accurate vehicle localization. Most underwater vehicles use a combination of Doppler velocity log (DVL), inertial navigation system (INS) and ship-board super short base line (SSBL) or ultra short base line (USBL) for localization and navigation. Position estimates are typically made based on these sensor measurements through dead reckoning (DR) or the use of an extended Kalman filter (EKF). Simple DR suffers from cumulative drift in position, which is unbounded and is typically in the order of $5 \%$ of distance travelled [4]. For a mission where a vehicle travels several kilometres, we would expect position uncertainty of several tens of metres. While the use of an EKF can bound estimates of position uncertainty, a shipboard SSBL typically has an uncertainty of $1 \%$ of the slant range, which for a distance of thousands of meters would be in the order of tens of metres. Clearly, these uncertainties in localization are significantly large compared

*Current address for Blair Thornton. Southampton Marine and Maritime Institute, University of Southampton, Southampton SO16 7QF, UK B.Thornton@soton.ac.uk to the mapping resolutions that can be achieved, and form a major bottleneck in our ability to perform meaningful highresolution studies of the seafloor.

State of the art position estimation solutions use a wider sensor suite, such as in [5], where a USBL provides $x-y$ absolute position, together with a Visual SLAM solution. Both position estimates are then fused in a parallel EKF solution with a DVL, IMU, GPS and a pressure sensor. The implementation shows that its accuracy is improved with respect to DR.

Visual SLAM enhances the estimation by detecting loop closures between different passes over the same areas. Several approaches have been reported in the literature, as in [6], where a stereo pair is used to extract features from the images and find matching candidates for loop closures. By these corrections, drift can be minimized. However, when trying to apply these techniques to underwater imaging, one is faced with a number of challenges that do not occur, or to a lesser degree in images taken on land: (1) image features change strongly depending on the point of view, mostly due to low altitudes or to wide camera lenses, which makes it difficult to extract them robustly; (2) it is also affected by absorption and scattering in water, and requires a light source [7]; (3) featureless terrains cannot be easily mapped [8] and (4) changing features like moving flora or fauna can affect the posterior feature matching process. Bathymetric SLAM can overcome some of these problems by using only featureless registration and discarding moving objects by filtering or treating them as outliers.

In Roman and Singh [9] a bathymetric SLAM solution using multibeam acoustic data is presented with a range resolution of $4 \mathrm{~cm}$. Inglis et al. [10] presented a structured light bathymetric mapping algorithm that accounts for errors in the horizontal component of the robot's position estimate.

This article explores another way of estimating the pose, accounting for four degrees of freedom: three for translation and one for orientation, whilst at the same time building a bathymetric map of the seafloor using laser based structured light. Laser stripe systems address some of the optical imaging issues. As only monochromatic light is projected, a colour can be chosen that is absorbed minimally in water, and because 
only a small volume of water is illuminated, the effect of backscatter is much smaller compared to imaging systems using flashes or continuous lights. Furthermore, since the laser has a small footprint on the seafloor, the power consumption of laser-based systems is typically significantly smaller than the systems that uses flash or lights. This makes it possible for the underwater vehicle to acquire images from higher altitudes and to cruise at higher speeds than if conventional lighting and imaging is used.

Structured light imaging provides 3D measurements of the seafloor with higher resolution than stereoscopy or multibeam sonar [11]. However, this system cannot exploit image features for SLAM in a conventional manner. Other approaches, as submap bathymetric SLAM described in this article, can be used.

This article is structured as follows: in section II the structured light system and the submap generation are described, whilst in section III the pairwise registration of the reconstructed three-dimensional submaps is presented. Then in section IV a SLAM solution to optimize the pose and orientation of the submaps is introduced. Finally, in section $\mathrm{V}$ the experimental results are presented and in section VI conclusions are delivered.

\section{STRUCTURED LiGHT SYSTEM}

The structured light system is formed by an underwater camera and a line laser mounted on a rigid rig with know dimensions, which is attached to an underwater vehicle. The camera and laser require a translational offset perpendicular to the direction of the laser projection plane. Often a relative angular offset is introduced to optimize the range and resolution of the system [12]. The captured images of the laser line projection on the seafloor provide a simple but efficient way of computing the terrain profile by triangulating the light rays originated from the laser back to the camera.

Figure 1 shows the structure of the submap registration pipeline, which is explained in more detail in the following sections. From images and vehicle positions submaps are created. Using their known uncertainty and position a list of possible loop closure candidates is proposed and a registration is issued. If the submaps match correctly, a new constraint is added to a pose-based graph SLAM and the graph is optimized. The algorithm finishes when there are no more submap candidates.

\section{A. Laser stripe detection}

As a first step all images are corrected for lens distortion. In each rectified image the laser stripe is detected with subpixel resolution. Each column is convolved with a triangular kernel, and the row yielding the highest computed value, provided it is bigger than a minimum threshold, is considered the approximate location of the laser line. The centroid is then computed to determine the position of the laser point candidate with sub-pixel resolution. The candidates from all columns are then grouped into clusters. Candidates from neighbouring columns that are close to each others go into the same cluster;

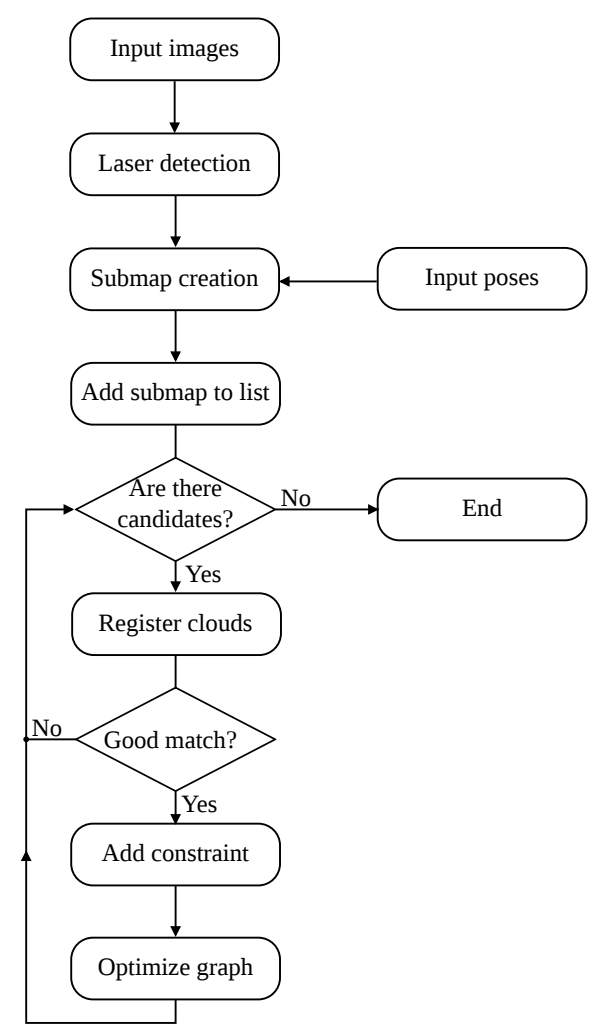

Figure 1. Submap registration pipeline.

if there is a jump in position between columns a new cluster starts. Laser line candidate points that are a part of large clusters are then passed on to the next step, while those that are in small clusters are considered outliers and are discarded.

The detected laser points $\boldsymbol{u}=\left(u_{i}, v_{i}\right) \in \mathbb{R}^{2}$ in $2 \mathrm{D}$ pixel coordinates are triangulated using the known rig dimensions and the camera calibration. In the camera coordinate system, the laser plane $\pi \in \mathbb{R}^{3}$ is known as

$$
\boldsymbol{\pi}: A x+B y+C z+D=0,
$$

where the coefficients $\{A, B, C, D\}$ are computed on a prior calibration stage. The three dimensional line that crosses the point $\boldsymbol{u} \in \mathbb{R}^{2}$ and the focal point of the camera is

$$
\boldsymbol{r}_{\boldsymbol{i}}(t):\left(\frac{u_{i}-c_{x}}{f_{x}} \cdot t, \frac{v_{i}-c_{y}}{f_{y}} \cdot t, t\right),
$$

where $\left(c_{x}, c_{y}\right)$ is the camera optical centre, $\left(f_{x}, f_{y}\right)$ is the focal length. These values can be obtained from the camera calibration.

The three dimensional laser point $\boldsymbol{m}_{\boldsymbol{i}} \in \mathbb{R}^{3}$ is therefore

$$
\boldsymbol{m}_{\boldsymbol{i}}=\boldsymbol{r}_{\boldsymbol{i}}(t) \cap \boldsymbol{\pi} .
$$

More information on how the laser detection and 3D point extraction works can be found in [13].

\section{B. Map generation}

In the laser stripe detection step the 3D points are computed in a reference frame tight to the vehicle. In order to obtain 
3D reconstructions in a global reference frame it is necessary to transform the coordinates, using the position of the vehicle when the image of the laser line was acquired. In our approach, an initial 6 degrees of freedom (DOF) estimate of the vehicle trajectory is obtained using the DR solution based on the measurements of a DVL, an IMU and a pressure sensor. It is straightforward to compound the laser points in a global reference frame given the position estimate of the vehicle: let $\boldsymbol{m}_{\boldsymbol{i}}=\mathcal{N}\left(\hat{\boldsymbol{m}}_{\boldsymbol{i}}, \boldsymbol{P}_{\boldsymbol{m}_{\boldsymbol{i}}}\right)$ be a triangulated 3D point in the expressed in the reference frame of the vehicle and $\boldsymbol{x}_{\boldsymbol{i}}=$ $\mathcal{N}\left(\hat{\boldsymbol{x}}_{\boldsymbol{i}}, \boldsymbol{P}_{\boldsymbol{x}_{\boldsymbol{i}}}\right)$ be the pose of the vehicle at the time the image was acquired. Then the position of one point $\boldsymbol{p}_{\boldsymbol{i}}=\mathcal{N}\left(\hat{\boldsymbol{p}}_{\boldsymbol{i}}, \boldsymbol{P}_{\boldsymbol{p}_{\boldsymbol{i}}}\right)$ of the submap can be computed as:

$$
\begin{aligned}
\hat{\boldsymbol{p}}_{\boldsymbol{i}} & =\hat{\boldsymbol{x}}_{i} \oplus \hat{\boldsymbol{m}}_{\boldsymbol{i}} \\
\boldsymbol{P}_{\boldsymbol{p}_{\boldsymbol{i}}} & =\boldsymbol{J}_{\mathbf{1} \oplus} \boldsymbol{P}_{\boldsymbol{x}_{\boldsymbol{i}}} \boldsymbol{J}_{\mathbf{1} \oplus}^{\top}+\boldsymbol{J}_{\mathbf{2} \oplus} \boldsymbol{P}_{\boldsymbol{m}_{\boldsymbol{i}}} \boldsymbol{J}_{\mathbf{2} \oplus}^{\top}
\end{aligned}
$$

where $\boldsymbol{J}_{\oplus}=\left[\boldsymbol{J}_{\mathbf{1} \oplus}, \boldsymbol{J}_{\mathbf{2} \oplus}\right]$ are the left and right halves $(6 \times 6)$ of the compounding Jacobian $(6 \times 12)$ [14].

\section{Submap sizing}

The main assumption of this algorithm is that the accuracy of the DR in a submap is consistent enough that drift can be neglected. This is reasonable because the time interval for each submap is set at a maximum of $30 \mathrm{~s}$, which for a mapping velocity of $0,4 \mathrm{~m} / \mathrm{s}$ would result in a position uncertainty of approximately $5 \mathrm{~mm}$. However, for the entire map the DR error would grow unbounded (or up to the constraints of SSBL or USBL uncertainty) and the complete map would appear distorted and in the case of overlapping transects, inconsistent. In order to produce self-consistent maps we developed an algorithm, explained in detail in the following sections, which registers submaps to each others to determine and compensate for the position estimation drift. Therefore, when aggregating the $3 \mathrm{D}$ points from the laser scanning step, we break them into a set of smaller submaps. The selection of the break point has to take into account the following: (1) the submap will be considered rigid, therefore it has to be small enough so that the error committed by compounding DR navigation is negligible, and (2) the submap has to be large enough to contain sufficient $3 \mathrm{D}$ information to be unambiguously registered to another submap.

In order to achieve this, the following conditions are applied to divide the data into submaps: (1) when the localization uncertainty of the last added line is bigger than a threshold, (2) when there is a jump in navigation, and (3) when the submap is considered too big. Once the submap is closed, all the $\boldsymbol{p}_{\boldsymbol{i}}$ points are referenced to a new coordinate frame coincident with the vehicle pose in the middle of the submap sequence and the position uncertainty at this point is applied to the submap. [9].

The submap points are triangulated using a Greedy Stepwise triangulation algorithm in two dimensional space (e.g. x-y), and are randomly sampled. At the same time the number of points can be reduced to speed up the subsequent processing steps. Each random sample is converted to a $3 \mathrm{D}$ point where its $z$ coordinate is interpolated using the tree points that form the two dimensional triangle.

\section{BOUNDED PAIRWISE REGISTRATION}

Once all the submaps are generated, loop closure candidates are proposed. These candidates are submaps whose projection into the $x-y$ plane, augmented by their uncertainty, overlap. For each proposed link, a pairwise registration is attempted to produce a 4 DOF relative pose transformation between the submaps. When computing the new estimate, only the position and the heading are adapted, as it is assumed that the roll and pitch measurements from the vehicle are accurate. The search space of the relative transformation is also bounded to the uncertainty of the position and heading of the current submap.

The registration presented in this paper is an optimization problem that estimates a rigid transformation. We assume: (1) the transformation is global (the same for every point within a submap), (2) can be uniquely defined by three noncollinear pairs of correspondences and (3) has 6 DoF. The constraints are found using the closest point criterion (CRC), which chooses the closest point as the match for the next iteration.

Let $\boldsymbol{P}_{\boldsymbol{a}}=\left\{p_{1}^{a}, \ldots, p_{n}^{a}\right\}$ and $\boldsymbol{P}_{\boldsymbol{b}}=\left\{p_{1}^{b}, \ldots, p_{m}^{b}\right\}$ be two overlapping submaps created with the previous assumptions. Let $\boldsymbol{p}_{\boldsymbol{i}}^{\boldsymbol{a}} \in \boldsymbol{P}_{\boldsymbol{a}}$ be a point in the submap $\boldsymbol{P}_{\boldsymbol{a}}$, and $\boldsymbol{p}_{j}^{\boldsymbol{b}}$ the closest point that lies in the plane defined by the three nearest neighbours of $\boldsymbol{p}_{i}^{\boldsymbol{a}}$ in $\boldsymbol{P}_{\boldsymbol{b}}$.

The function to minimize depends on the L2 norm and the $z$ distance between the points $\boldsymbol{p}_{\boldsymbol{i}}^{\boldsymbol{a}}$ and $\boldsymbol{p}_{j}^{\boldsymbol{b}}$ subject to small translations and heading. The parameter block can be simplified to $4 \operatorname{DoF} \boldsymbol{x}=\{\Delta x, \Delta y, \Delta z, \Delta \theta\}$ given our application. Common ROVs or surveying AUVs for imagery have pitch and roll measurements with tightly bounded uncertainties, so it is assumed that these degrees are sufficiently accurate in the for the purpose of this study. Furthermore, vehicles are normally not controlled in these DoF, and it is unlikely that the bias error significantly drift over time.

The minimization function can be expressed as

$$
\begin{array}{ll}
\underset{\boldsymbol{x}}{\operatorname{minimize}} & \frac{1}{N} \sum_{i=1}^{N} f\left(\boldsymbol{T}(\boldsymbol{x}) \cdot \boldsymbol{p}_{i}^{a}, \boldsymbol{q}_{i}^{b}\right)^{2}, \\
\text { subject to } & \boldsymbol{x}_{\min } \leq \boldsymbol{x} \leq \boldsymbol{x}_{\max },
\end{array}
$$

where $\boldsymbol{T}(\boldsymbol{x})$ is the $4 \times 4$ homogeneous matrix that depends on the parameter block. The final value of the (6) is the cost, and the transformation is the output of the minimization algorithm, and will be added to a pose-based graph SLAM as a new edge if the resulting minimization cost is below a maximum allowable error. $\boldsymbol{x}_{\min }$ and $\boldsymbol{x}_{\max }$ bound the argument search space. These terms depend directly on the position uncertainty of the submaps being registered. The function $f\left(\boldsymbol{p}_{i}^{a}, \boldsymbol{q}_{i}^{b}\right), \boldsymbol{p}_{i}^{a} \in \boldsymbol{P}_{\boldsymbol{a}}, \boldsymbol{q}_{i}^{b} \in \boldsymbol{P}_{\boldsymbol{b}}$ is defined as

$$
f\left(\boldsymbol{p}_{i}^{a}, \boldsymbol{q}_{i}^{b}\right)= \begin{cases}e_{z} & \text { if } e<e_{\text {min }} \\ e_{z}+e_{l_{2}} & \text { if } e \geq e_{\text {min }}\end{cases}
$$




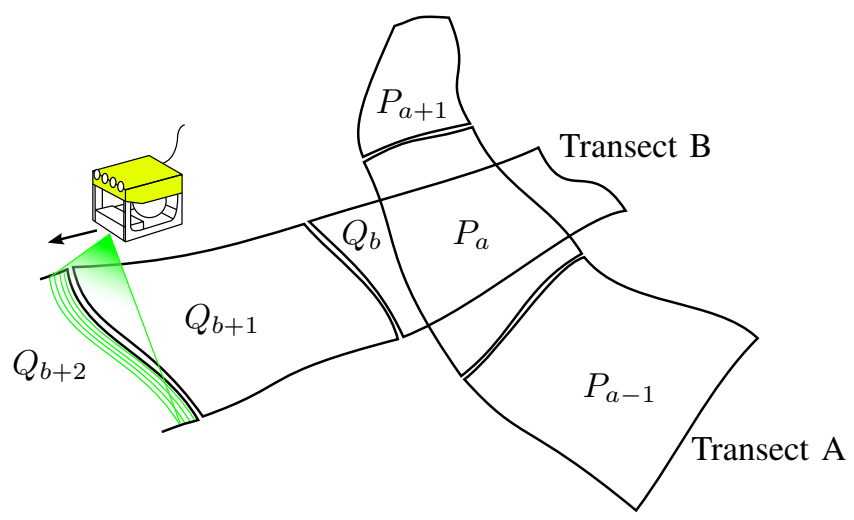

Figure 2. An underwater vehicle scans the seafloor using a laser stripe. Each submap is stored with known transformations and uncertainty for posterior optimization.

where $e_{z}$ is the distance in meters of the point $\boldsymbol{p}_{i}^{a}$ to its projection $\boldsymbol{q}_{i}^{b}$ on the plane formed by the three closest points in a $\mathrm{x}-\mathrm{y}$ projected two-dimensional cloud. The term $e_{l_{2}}$ is the euclidean distance of the point $\boldsymbol{p}_{i}^{a}$ to the closest point in $\boldsymbol{P}_{b}$ after the transformation. Finally, the term $e_{\min }$ switches between these two errors and depends on the random sampled submap resolution. It is used to avoid over-fitting the submaps to the sampled points or to the noise.

Figure 2 shows an example of two intersecting transects. Submaps $\boldsymbol{P}_{\boldsymbol{a}}$ from transect A and $\boldsymbol{P}_{\boldsymbol{b}}$ from transect B overlap within an uncertainty determined search space, so a relative transformation between them can be computed using the registration algorithm of the presented solution.

\section{BATHYMETRIC SLAM}

Most common SLAM solutions have two clear parts: (1) a front-end and (2) a back-end [15]. In this approach, the frontend is the pairwise registration explained in section III, and the back-end is a pose-based graph SLAM explained below.

A pose-based graph SLAM is a graphical representation of the SLAM optimization problem where poses are represented as nodes and relative transformations as edges in a graph. Let $\boldsymbol{x}=\left(\boldsymbol{x}_{1}, \ldots, \boldsymbol{x}_{T}\right)^{\top}$ be a vector of parameters, where $\boldsymbol{x}_{i}$ describes the pose of node $i$. Let $\boldsymbol{z}_{i j}$ and $\boldsymbol{\Omega}_{i j}$ be the mean and the information matrix of a measurement between the node $i$ and the node $j$. This measurement is a transformation that makes the observations obtained from $i$ maximally overlap with the ones acquired from $j$. Let $\hat{\boldsymbol{z}}_{i j}\left(\boldsymbol{x}_{i}, \boldsymbol{x}_{j}\right)$ be the measurement between nodes $i$ and $j$, which usually is the relative transformation between these two. The log-likelihood $l_{i j}$ of a measurement $z_{i j}$ is

$$
\boldsymbol{l}_{i j} \propto \boldsymbol{e}_{i j}^{\top} \boldsymbol{\Omega}_{i j} \boldsymbol{e}_{i j}
$$

where $\boldsymbol{e}_{i j}=\boldsymbol{e}_{i j}\left(x_{i}, x_{j}\right)=\boldsymbol{z}_{i j}-\hat{\boldsymbol{z}}_{i j}\left(\boldsymbol{x}_{i}, \boldsymbol{x}_{j}\right)$ is the error function that computes the difference between the expected and the real observation. For simplicity, the indices of the measurements have been encoded in the indices of the error function. The equation to minimize is the negative $\log _{-}$

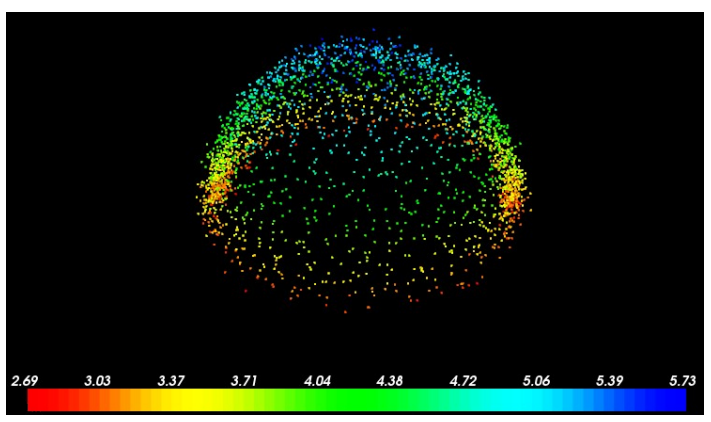

(a) Hemisphere generated with a standard deviation of $0.1 \mathrm{~m}$

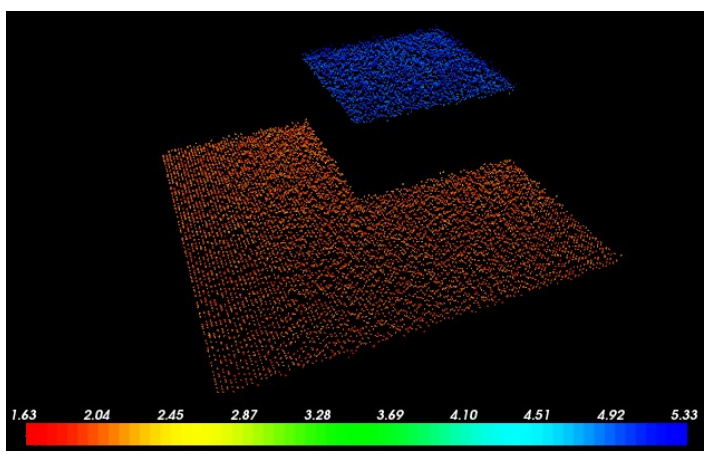

(b) Step generated with a standard deviation of $0.1 \mathrm{~m}$

Figure 3. Computer generated data to test the registration algorithm.

likelihood $l_{i j}$ to achieve the goal of a maximum likelihood approach.

$$
\boldsymbol{x}^{*}=\underset{\boldsymbol{x}}{\arg \min } \sum_{(i, j) \in \mathcal{C}} \boldsymbol{e}_{i j}^{\top} \boldsymbol{\Omega}_{i j} \boldsymbol{e}_{i j}
$$

The output of this equation are the optimized relative transformation encoded in the edges of the pose-based graph SLAM.

\section{EXPERIMENTAL RESULTS}

Two sets of experiments have been conducted to evaluate the pairwise registration, one with computer generated data to evaluate the results of the registration algorithm, and another with underwater imagery gathered during a research cruise in the Iheya North field of the Okinawa Trough. The SLAM results will be presented in an upcoming paper.

The registration has been implemented using Ceres library [16], and the graph SLAM using g2o library [17], both in $\mathrm{C}++$. The code is available for testing at [18].

\section{A. Evaluation}

Two different point cloud sets have been generated to assess the accuracy of the registration. The first one is a set of 3D points sampled from a hemisphere with a radius of $5 \mathrm{~m}$, and the second is a sharp step of $3 \mathrm{~m}$ over a $6 \times 6 \mathrm{~m}$ area.

Pairs of these clouds were generated using different sampling noises as shown in figure 3 and at different locations in space. The artificially introduced noise has zero mean and its standard deviation was increased in the following manner: $0.01,0.1,0.2,0.3,0.4,0.5$ and $1 \mathrm{~m}$. The relative 
transformation between the submap pair has been changed from $1,2,4$ and $6 m$ for $x$ and $y$ coordinates, and three different $z$ distances have also been used: $0,0.5$ and $1 \mathrm{~m}$. The yaw orientation has been changed from 0 to 0.2 radians in 0.1 radian steps. The orientation alteration has not been introduced in the hemisphere dataset. This results in 336 different registration trials for the hemisphere and 1008 trials for the step dataset. These pairs were input to the algorithm, which computed the transformation to best align them. The difference of the computed transformation with compared to the actual transformation are presented in table I and in figure 4(a) for the hemisphere, and in table II and in figure 4(b) for the step. In the tables, the minimum, maximum, mean, median and standard deviation of the translation error (L2 norm) of the registration for all the different configurations are presented. Results on the orientation alignment for the step registration are also shown on table III.

For each trial the algorithm was only run once. Different runs may end up in slightly different results, since the random sampling makes the submaps look different, but on average it does not change the outcome significantly.

\section{B. Seafloor data}

In 2015 an underwater survey was carried out with the ROV Hyper-Dolpin (JAMSTEC) at the Iheya North Field, Okinawa, Japan. The vehicle was equipped with a monochrome camera and a laser stripe. The setup used was similar to the one presented in [19]. A large area of seafloor, including a $30 \mathrm{~m}$ tall mound and an artificial vent, was mapped from an altitude of approximately $8 \mathrm{~m}$.

Three passes over the artificial vent that were made during that survey have been used to test the registration algorithm. The three transects and the generated graph can be seen in figures 5(a) and 5(b) respectively. The transects A, B and C are formed by submap 0 to 2, 3 to 6 and 7 to 11 . These submaps where split evenly at $34 \mathrm{~min} 23.91 \mathrm{sec}, 61 \mathrm{~min} 29.60 \mathrm{sec}$ and $345 \mathrm{~min} 41.35 \mathrm{sec}$ from the start of the dive. Each transect duration is 220 seconds.

The graph shows the submaps as nodes and their relative transformations as edges, where dashed lines are loop closure candidates.

Figure 6 shows submaps 6 (transect B) and 9 (transect C) generated using their initial pose estimates based on DR (subfigure 6(a)) and after (subfigure 6(b)) the registration algorithm was applied. It is worth to notice that the output of the algorithm is a corrective translation of $(-1.297,0.209,0.238) \mathrm{m}$ and a heading of $0.031 \mathrm{rad}$ between the known relative transformation and the corrected registration. Registering these two submaps took 52 iterations and 3.49 seconds on a Intel $i 5$ processor at $2.4 \mathrm{GHz}$ with $8 \mathrm{~Gb}$ of RAM. The point density has changed due to the random subsampling used in the first step of the registration algorithm.

\section{CONCLUSIONS AND Future WORK}

A pipeline to improve the accuracy of high-resolution seafloor bathymetry using SLAM has been presented and its registration algorithm evaluated. This same pipeline could also work with other sensor output streams, such as point clouds from stereo cameras or from other structured light sensors.

In the evaluation of the computer generated data, we could see that lower noises gave the most stable output, with most of the outliers when the two submaps where initially separated by a large distance, and also when the orientation offset was at its maximum value. The reason for this is that the submap transformation output fall into local minima away from the true solution. This means that the input reconstruction must be as accurate as possible, as it is the starting point for the registration. Regarding the orientation misalignment, the differences in the registration results (e.g. error) do not show any strong trend depending on which was the starting orientation. This solution is able to cope with small misalignments in orientation (up to $0.2 \mathrm{rad}$ ). For noisy data, the features of the submap (e.g. the step or the smoothed surface of the hemisphere) to register ended up over-fitting the submaps, registering to noise in a local minima configuration, whilst for the step, the minimization function forces the submaps to better align. The results start to have higher errors when the noise is comparable with the step itself ( $3 \mathrm{~m}$ step vs a standard deviation of $0.5-1.0 \mathrm{~m}$ ). From these results it is clear that the registration works well with noisy data, and it is able to cope with up to one-to-two meter misalignments if the overlap is big enough.

Regarding results with seafloor data, the DR and position uncertainty played a significant role in the results. Getting a good initial position estimate is crucial for the success of the pipeline, since terrain-based registrations, such as ICP, are prone to fall in local minima while performing the minimization step.

Future work will be focused in further studying these results, and the sources of noise, such as the camera calibration, the laser transformation, the laser detection in the image and the robot position accuracy and uncertainty, and implementation of methods to improve robustness to local minima.

Also, further results in the evaluation of the SLAM solution will be presented, together with a parametrization study on the submap size, or whether smoothing out the submaps prior to registration, or using multiscale resolution as suggested in [20] is applicable.

\section{ACKNOWLEDGMENTS}

This collaborative research work between the University of the Balearic Islands and the University of Tokyo has been partially supported by grant BES-2012-054352 (FPI), TIN2014-58662-R (ARSEA) and DPI2014-57746-C32-R (SUPERION), by the Spanish Ministry of Economy and Competitiveness and ERDF Funding.

\section{REFERENCES}

[1] B. Thornton, A. Bodenmann, O. Pizarro, S. B. Williams, A. Friedman, R. Nakajima, K. Takai, K. Motoki, T.-o. Watsuji, H. Hirayama, Y. Matsui, H. Watanabe, and T. Ura, "Biometric Assessment of Deepsea Vent Megabenthic Communities using Multi- Resolution 3D Image Reconstructions," Deep-sea Research Part 1: Oceanographic Research Papers, vol. (accepted), 2014. 
Table I

ERROR (M) VS NOISE (M) FOR THE HEMISPHERE REGISTRATION.

\begin{tabular}{rccccccc}
\hline Noise (m) & $\mathbf{0 . 0 1}$ & $\mathbf{0 . 1}$ & $\mathbf{0 . 2}$ & $\mathbf{0 . 3}$ & $\mathbf{0 . 4}$ & $\mathbf{0 . 5}$ \\
\hline $\min (\mathrm{m})$ & $2.9808 \cdot 10^{-5}$ & $1.2830 \cdot 10^{-4}$ & $4.5229 \cdot 10^{-4}$ & $8.1310 \cdot 10^{-3}$ & $3.3792 \cdot 10^{-2}$ & $1.6480 \cdot 10^{-1}$ & $1.9118 \cdot 10^{-1}$ \\
$\operatorname{median}(\mathrm{m})$ & $6.5197 \cdot 10^{-4}$ & $6.7492 \cdot 10^{-3}$ & $3.4790 \cdot 10^{-2}$ & $1.2533 \cdot 10^{-1}$ & $2.9952 \cdot 10^{-1}$ & $6.4865 \cdot 10^{-1}$ & 2.2831 \\
$\max (\mathrm{m})$ & $2.5803 \cdot 10^{-3}$ & $3.1486 \cdot 10^{-2}$ & $1.3051 \cdot 10^{-1}$ & $2.2717 \cdot 10^{-1}$ & 3.7970 & 3.6060 \\
stdev (m) & $6.1572 \cdot 10^{-4}$ & $7.6254 \cdot 10^{-3}$ & $3.1734 \cdot 10^{-2}$ & $4.8096 \cdot 10^{-2}$ & $4.2485 \cdot 10^{-1}$ & $6.0853 \cdot 10^{-1}$ & $7.4281 \cdot 10^{-1}$ \\
mean (m) & $8.4171 \cdot 10^{-4}$ & $9.3578 \cdot 10^{-3}$ & $4.0890 \cdot 10^{-2}$ & $1.2240 \cdot 10^{-1}$ & $3.4668 \cdot 10^{-1}$ & $7.4070 \cdot 10^{-1}$ & 2.0858 \\
\hline
\end{tabular}

Table II

ERROR (M) VS NOISE (M) FOR THE STEP REGISTRATION.

\begin{tabular}{|c|c|c|c|c|c|c|c|}
\hline Noise (m) & 0.01 & 0.1 & 0.2 & 0.3 & 0.4 & 0.5 & 1 \\
\hline $\min (\mathrm{m})$ & $1.1358 \cdot 10^{-11}$ & $3.0263 \cdot 10^{-11}$ & $4.1608 \cdot 10^{-11}$ & $3.5444 \cdot 10^{-11}$ & $1.5265 \cdot 10^{-10}$ & $2.3644 \cdot 10^{-3}$ & $5.0385 \cdot 10^{-1}$ \\
\hline median (m) & $6.6467 \cdot 10^{-3}$ & $3.4195 \cdot 10^{-3}$ & $2.6962 \cdot 10^{-3}$ & $1.5203 \cdot 10^{-3}$ & $3.0901 \cdot 10^{-3}$ & $4.2186 \cdot 10^{-1}$ & 1.5437 \\
\hline $\max (\mathrm{m})$ & $1.8029 \cdot 10^{-2}$ & $2.0999 \cdot 10^{-2}$ & $2.6460 \cdot 10^{-1}$ & $9.0549 \cdot 10^{-1}$ & $8.4276 \cdot 10^{-1}$ & 2.2338 & 2.3790 \\
\hline stdev (m) & $5.6933 \cdot 10^{-3}$ & $5.2982 \cdot 10^{-3}$ & $2.2064 \cdot 10^{-2}$ & $1.1568 \cdot 10^{-1}$ & $2.2338 \cdot 10^{-1}$ & $3.4039 \cdot 10^{-1}$ & $3.7719 \cdot 10^{-1}$ \\
\hline mean $(\mathrm{m})$ & $6.4811 \cdot 10^{-3}$ & $4.7769 \cdot 10^{-3}$ & $5.2631 \cdot 10^{-3}$ & $2.4966 \cdot 10^{-2}$ & $1.1199 \cdot 10^{-1}$ & $3.8441 \cdot 10^{-1}$ & 1.5345 \\
\hline
\end{tabular}

Table III

ERROR (M) VS INITIAL ORIENTATION (M) FOR THE STEP REGISTRATION.

\begin{tabular}{rccc}
\hline Orientation (rad) & $\mathbf{0}$ & $\mathbf{0 . 1}$ & $\mathbf{0 . 2}$ \\
\hline $\min (\mathrm{m})$ & $1.1358 \cdot 10^{-11}$ & $4.9450 \cdot 10^{-5}$ & $3.5752 \cdot 10^{-5}$ \\
$\operatorname{median}(\mathrm{m})$ & $5.5470 \cdot 10^{-6}$ & $1.4234 \cdot 10^{-2}$ & $1.0577 \cdot 10^{-2}$ \\
$\max (\mathrm{m})$ & 5.0827 & 5.6844 & 5.8880 \\
$\operatorname{stdev}(\mathrm{m})$ & $9.1019 \cdot 10^{-1}$ & 1.0064 & 1.1020 \\
$\operatorname{mean}(\mathrm{m})$ & $4.8451 \cdot 10^{-1}$ & $6.1748 \cdot 10^{-1}$ & $6.3240 \cdot 10^{-1}$ \\
\hline
\end{tabular}

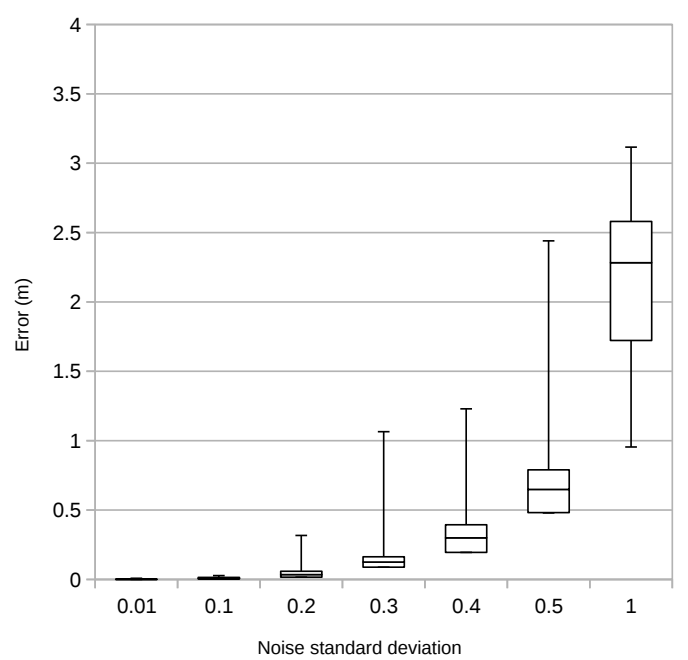

(a) Hemisphere registration

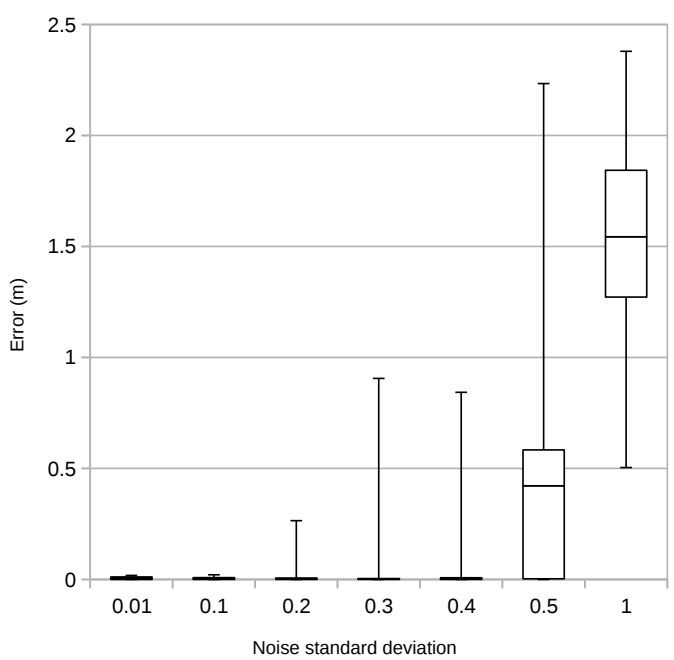

(b) Step registration

Figure 4. Box plot of error vs noise standard deviation 


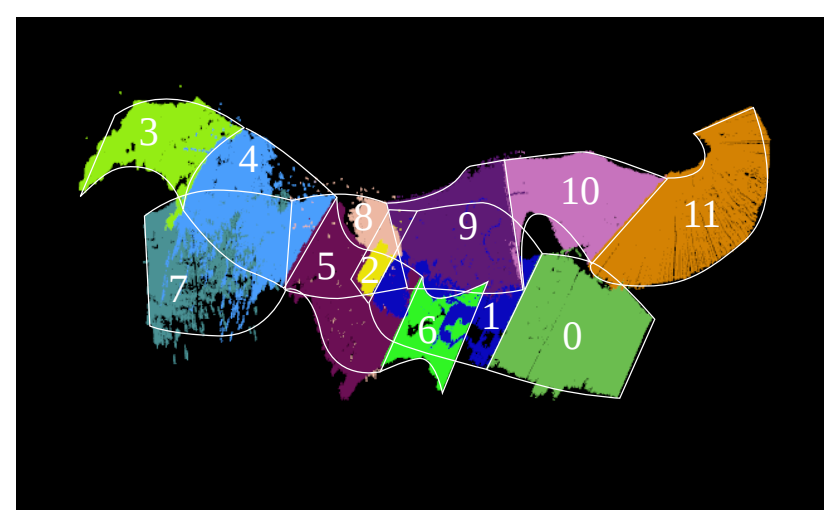

(a) Transects $\mathrm{A}, \mathrm{B}$ and $\mathrm{C}$ formed by submaps $0-2,3-6$ and 7-11 respectively

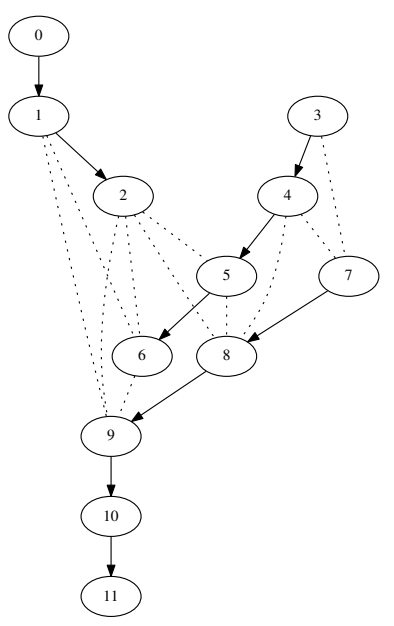

(b) Resulting graph

Figure 5. Three 3D reconstructed transects over an artificial vent

[2] N. Gracias, P. Ridao, R. Garcia, J. Escartín, M. L’Hour, F. Cibecchini, R. Campos, M. Carreras, D. Ribas, N. Palomeras, L. Magi, A. Palomer, T. Nicosevici, R. Prados, R. Hegedüs, L. Neumann, F. de Filippo, and A. Mallios, "Mapping the moon: Using a lightweight auv to survey the site of the 17th century ship La Lune," in OCEANS - Bergen, 2013 MTS/IEEE, June 2013, pp. 1-8.

[3] M. Massot-Campos and G. Oliver-Codina, "Underwater laser-based structured light system for one-shot 3d reconstruction," in IEEE SENSORS 2014 Proceedings, Nov 2014, pp. 1138-1141.

[4] M. Hammond and S. M. Rock, "A SLAM-based approach for underwater mapping using AUVs with poor inertial information," 2014 IEEE/OES Autonomous Underwater Vehicles, AUV 2014, 2014.

[5] E. Guerrero Font, M. Massot Campos, P. L. Negre Carrasco, F. BoninFont, and G. Oliver Codina, "A USBL-Aided Multisensor Navigation System for Field AUVs," in 2016 IEEE International Conference on Multisensor Fusion and Integration for Intelligent Systems (MFI). IEEE, sep 2016.

[6] F. Guth, L. Silveira, S. Botelho, P. Drews, and P. Ballester, "Underwater SLAM: Challenges, state of the art, algorithms and a new biologicallyinspired approach," in 5th IEEE RAS/EMBS International Conference on Biomedical Robotics and Biomechatronics. IEEE, aug 2014, pp. 981-986.

[7] F. Bonin-Font and A. Burguera, "Imaging systems for advanced underwater vehicles," Journal of Maritime Research, vol. VIII, no. 1, pp. 65-86, 2011

[8] S. Barkby, S. B. Williams, O. Pizarro, and M. V. Jakuba, "A feature-

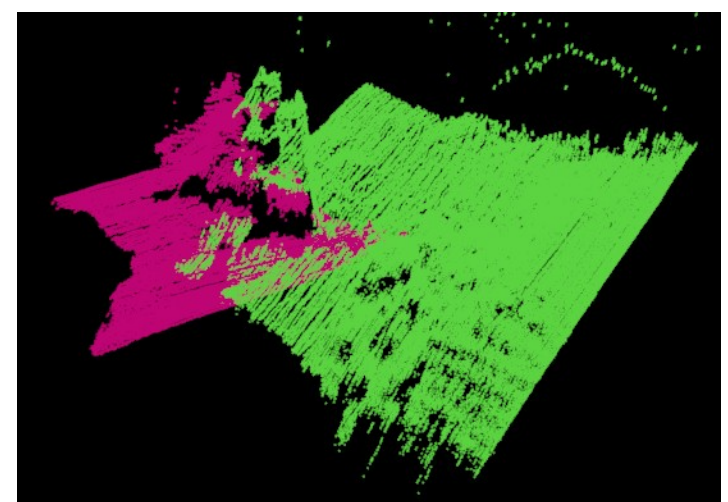

(a) Original submap of the artificial vent with DR navigation

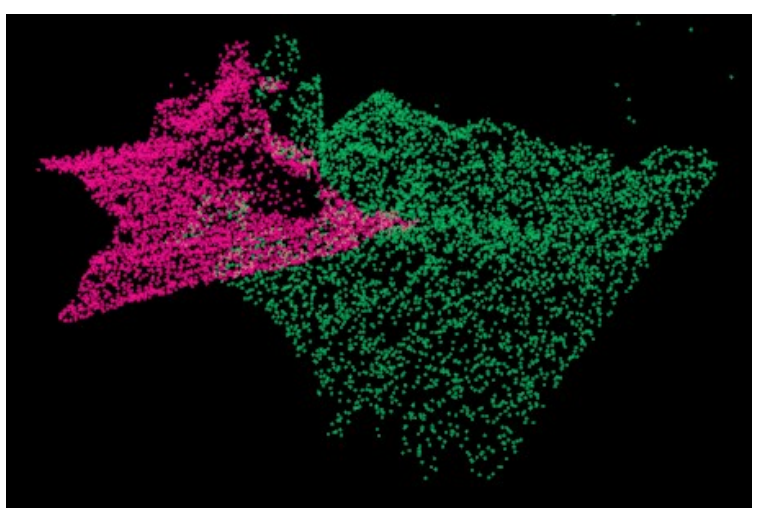

(b) Registered \& random sampled submaps

Figure 6. Registration example of an underwater human made structure

less approach to efficient bathymetric SLAM using distributed particle mapping," Journal of Field Robotics, vol. 28, no. 1, pp. 19-39, jan 2011.

[9] C. Roman and H. Singh, "A Self-Consistent Bathymetric Mapping Algorithm," Journal of Field Robotics, vol. 24, no. 1-2, pp. 23-50, jan 2007.

[10] G. Inglis, C. Smart, I. Vaughn, and C. Roman, "A pipeline for structured light bathymetric mapping," in 2012 IEEE/RSJ International Conference on Intelligent Robots and Systems. IEEE, oct 2012, pp. 4425-4432.

[11] M. Massot, G. Oliver, H. Kemal, Y. Petillot, and F. Bonin-Font, "Structured light and stereo vision for underwater 3D reconstruction," in IEEE/MTS Oceans'15, 2015.

[12] A. Bodenmann, B. Thornton, and T. Ura, "3d mapping of the seafloor in color using a single camera," Sea Technology, vol. 51, no. 12, pp. $51-53,2010$.

[13] _ "Generation of high-resolution 3d reconstructions of the seafloor in colour using a single camera and structured light," Journal of Field Robotics, vol. (accepted), 2016.

[14] R. Smith, M. Self, and P. Cheeseman, "Estimating uncertain spatia relationships in robotics," in Robotics and Automation. Proceedings. 1987 IEEE International Conference on, vol. 4, Mar 1987, pp. 850850.

[15] G. Grisetti, R. Kuemmerle, C. Stachniss, and W. Burgard, "A tutorial on graph-based SLAM," Intelligent Transportation Systems Magazine, IEEE, vol. 2, no. 4, pp. 31-43, 2010.

[16] S. Agarwal, K. Mierle, and Others, "Ceres solver," http://ceres-solver org.

[17] R. Kummerle, G. Grisetti, H. Strasdat, K. Konolige, and W. Burgard, "G2o: A general framework for graph optimization," in 2011 IEEE International Conference on Robotics and Automation. IEEE, may 2011, pp. 3607-3613.

[18] “Terrain slam,” https://github.com/srv/terrain_slam, accessed: 2016-0824

[19] A. Bodenmann, B. Thornton, and T. Ura, "Visual 3D mapping to 
measure hydrothermal deposit growth rates at a man-made deep sea vent," in Proc. of IEEE/OES AUV 2014. Oxford, MS: IEEE, oct 2014.

[20] G. K. L. Tam, Z. Q. Cheng, Y. K. Lai, F. C. Langbein, Y. Liu,

D. Marshall, R. R. Martin, X. F. Sun, and P. L. Rosin, "Registration of 3d point clouds and meshes: A survey from rigid to Nonrigid," IEEE Transactions on Visualization and Computer Graphics, vol. 19, no. 7, pp. 1199-1217, 2013. 